\title{
IgE antibody responses to platinum group metals: a large scale refinery survey
}

\author{
R D MURDOCH, ${ }^{1}$ J PEPYS, ${ }^{1}$ AND E G HUGHES ${ }^{2}$ \\ From the Department of Medicine, ${ }^{1}$ Guy's Hospital Medical School, London SE1 9RT, and Johnson Matthey, ${ }^{2}$ \\ Southgate, London N146ET, UK
}

ABSTRACT All 306 South African platinum refinery workers (116 white, 190 coloured) accepted for employment on grounds of absence of evidence of atopy were investigated using the skin prick test and RAST to detect sensitivity to platinum, palladium, and rhodium salts. RAST studies were made for these, together with HSA and DNP-HSA RAST. Of the 306 workers, 38 had a positive skin prick test to the platinum halide salts; of these, one gave a positive reaction to the palladium salt and six to the rhodium salt. There were no isolated positives to the rhodium and palladium halide salts. Total IgE levels were raised in 24 of the $38(63 \%)$ platinum salt prick test positive workers compared with only 43 of the $268(16 \%)$ prick test negative group $(p<0.001)$. Positive RASTs were obtained in $62 \%$ of those with positive skin tests to the platinum salts. Four of the six giving positive rhodium salt skin tests gave a positive RAST to rhodium salt. Of these, two gave positive RASTS to HSA and all four to DNP-HSA. The palladium salt RAST was negative in the single skin test reactor. In the platinum salt skin test positive group a raised HSA RAST was obtained in $10.5 \%$ compared with only $2.5 \%$ in the skin negative group. Twenty one per cent of the platinum salt skin positive group had a raised RAST score to DNP-HSA with only $3.5 \%(4 / 116)$ in the skin test negative group, of whom three also had a raised HSA RAST. The latter findings are suggestive of IgE antibody production to new antigenic determinants in HSA produced by conjugation with the platinum salts.

Ammonium tetrachloroplatinite II $\left[\left(\mathrm{NH}_{4}\right)_{2} \mathrm{PtCl}_{4}\right]$ ammonium hexachloroplatinate IV $\left[\left(\mathrm{NH}_{4}\right)_{2} \mathrm{PtCl}_{6}\right]$ have been implicated as the main occupational sensitising agents among platinum refinery workers and both are capable of inducing and eliciting type 1 hypersensitivity in exposed workers. ${ }^{1-3}$ Little is known, however, concerning the sensitising capacity or indeed the cross reactivity between the halide salts of platinum and the other platinum group metal salts such as palladium and rhodium. During the time at work, platinum refinery process workers are known to be exposed to comparable levels of the salts of palladium and rhodium.

There are some reports of sensitivity to palladium and palladium salts. ${ }^{4-6}$ Most of the reports, however, are concerned with contact dermatitis, a type IV reaction, whereas allergy to the platinum halide salts is predominantly a type I reaction. Reports of rhodium salt sensitisation per se are much less common (G M Levene, personal communication).

The sensitising and eliciting capacity of these metal

Accepted 13 May 1985 salts was studied in a large platinum refinery where workers with positive skin prick tests to the salts of platinum and other platinum group metal salts were found. It should be noted, however, that in no cases were positive reactions found in the absence of a positive platinum halide salt skin test.

In occupational allergic disease there may be an appreciable cross reactivity between closely related analogues of the principle sensitising/eliciting agent. This is especially true of the acid anhydrides, phthalic, trimellitic, and tetrachlorophthalic ${ }^{78}$ and the isocyanates, toluene diisocyanate and its analogues pTMI, MDI, and HDI. ${ }^{9}$

It is also possible that antibodies directed against the probable carrier molecule, human serum albumin (HSA), HSA in partially denatured form, or both, may be induced by the effects of the hapten conjugation in vivo. This has been shown in other compounds where new antigenic determinants (NADS) may be formed after hapten binding with TDI. ${ }^{10}$ Furthermore, in subjects sensitive to nickel antibodies have been directed against Ni-HSA fragments. ${ }^{112}$

The aims of this study, therefore, were to 
investigate the prevalence of platinum salt allergy, and of antibody directed against other platinum group metal salts to determine the presence of antibody to HSA and antigenically denatured HSADNP, to study their relations, and to correlate these findings with IgE levels.

\section{Materials and methods}

\section{SUBJECTS INVESTIGATED}

Sera were collected from 306 South African platinum refinery workers (116 white, 190 coloured) over three days. This represented $100 \%$ of the work force, all of whom had a negative history of allergic symptoms to common environmental allergens in their preemployment medical screening. (Atopic personnel are not employed because of their greater predisposition to develop platinum salt hypersensitivity.)

Eight cord blood sera were used for minimum nonspecific RAST binding (mean total $\mathrm{IgE} 0.5 \mathrm{IU} / \mathrm{ml}$ ).

Twenty sera from normal non-atopic white British blood donors (mean total IgE 30.4, range 2-158 $\mathrm{IU} / \mathrm{ml}$ ) were used for comparative purposes only in assessing total IgE levels.

Ten patients with Hodgkin's disease with raised total serum IgE levels (between 1750 and 22500 $\mathrm{IU} / \mathrm{ml}$ ) with no known specificity were used to determine the effects of high total IgE in the individual RAST systems.

\section{SKIN TESTS}

All 306 subjects were skin prick tested on the washed volar surface of the arm with ammonium hexachloroplatinate IV, sodium hexachloroplatinate, and sodium tetrachloroplatinite, ${ }^{3}$ dissolved freshly in Coca's fluid $(5.0 \mathrm{~g} \mathrm{NaCl}, 4.0 \mathrm{~g}$ phenol, and $2.75 \mathrm{~g} \mathrm{NaH}$ $\mathrm{CO}_{3}$ in $100 \mathrm{ml}$ distilled water). Tenfold dilutions were made from $10^{-3}$ to $10^{-9} \mathrm{~g} / \mathrm{ml}$. A positive reaction was taken to be greater than a $2 \mathrm{~mm}$ weal. All were also tested with the corresponding salts of palladium, rhodium, iridium, and ruthenium.

\section{PLATINUM GROUP METAL SALTS (PGM)}

The platinum group metal salts used in both skin prick test and in conjugate preparation were kindly supplied by Johnson Matthey Chemicals, Royston. All were of high grade spectroscopic purity.

TOTAL SERUM IgE DETERMINATIONS

IgE levels were measured by a direct solid phase radioimmunoassay (phadebas IgE PRIST, Pharmacia).
PLATINUM GROUP METAL SALT (PGM) RAST

Preparation of sepharose HSA-PGM

The solid phase preparations in all platinum group metal salt RAST systems used the method of Cromwell et al. ${ }^{13}$ Solid phase preparations consisted of $50 \mathrm{ml}$ packed volume of the sepharose-HSA complex to which was added $150 \mathrm{ml}, 0.33 \mathrm{mM}$ of either ammonium tetrachloroplatinite II, ammonium tetrachloropallidite II, or ammonium aquopentachlororhodite III in PBS without azide. The suspensions were mixed for three hours at room temperature followed by 18 hours at $4^{\circ} \mathrm{C}$. The solid phases were washed with 2 litre quantities of phosphate buffered saline and stored as a 1:4 suspension in phosphate buffered saline $(0.02 \%$ sodium azide and $0.5 \%$ normal horse serum (NHS).

\section{Sepharose-HSA preparation}

Coupling HSA to CNBr activated sepharose used the method of Cromwell et al with the exception that $5 \mathrm{mg}$ HSA (Sigma V) was coupled to $1 \mathrm{ml}$ packed volume rather than $10 \mathrm{mg} / \mathrm{ml}$ used by Cromwell et al ${ }^{13}$

\section{Sepharose HSA-DNP}

One hundred milligrams of HSA were dissolved in $5 \mathrm{ml}, 0.15 \mathrm{M}$ potassium carbonate, to which was added $20 \mathrm{mg}$ dinitrobenzene sulphonate, sodium salt, and mixed for 18 hours at $4^{\circ} \mathrm{C}$. The resulting conjugate was passed through a Sephadex G25 column. The conjugates were diluted 1:20 and the hapten loading on the carrier protein determined spectrophotometrically, at $280 \mathrm{~nm}$ and $360 \mathrm{~nm}$, allowing for DNP interference with the protein OD at $280 \mathrm{~nm} .{ }^{14}$ The conjugate contained 6.1 DNP/protein molecule. The protein-DNP conjugate was then coupled at $10 \mathrm{mg} / \mathrm{ml}$ to a Sepharose solid phase after the method of Cromwell et al. ${ }^{13}$

\section{RAST PROCEDURES}

Serum samples were tested blind in duplicate on one occasion by the addition of $50 \mu \mathrm{l}$ of neat serum to 100 $\mu 1$ 1:4 suspension of freshly washed solid phase in polystyrene tubes (Seward GW4). Tubes were sealed with plate sealing tape (Selotape 3M) and incubated for 18 hours at room temperature with horizontal shaking. The solid phase was washed three times with saline $\left(0.9 \% \mathrm{NaCl}, 0.02 \% \mathrm{NaN}_{3}, 0.5 \%\right.$ Tween 20 , $0.5 \%$ normal horse serum) by resuspension and centrifugation. Then $100 \mu \mathrm{l}^{125} \mathrm{I}$ labelled human anti-IgE (Pharmacia) was added and incubated for a further 18 hours at room temperature. Tubes were washed three times, as before, and counted for one minute on a gamma counter (Hydragamma, Innotron).

Sera for each RAST system were tested in one assay to eliminate interassay variation. Sera from cord blood, normal donors, patients with Hodgkin's 
disease, and known platinum positive controls were included in each assay. This allowed the determination of minimum baseline binding, maximum non-specific uptake, and assay reproducibility respectively. Sera giving RAST scores at or greater than unity in the platinum RAST were selected for further study.

\section{Results}

\section{SKIN PRICK TESTS}

All 306 subjects were skin prick tested to the salts of platinum palladium, rhodium iridium, and ruthenium and 38 gave reproducible positive Type 1 reactions to ammonium hexachloroplatinate, sodium hexachloroplatinate, and sodium tetrachloroplatinite at $10^{-3} \mathrm{~g} / \mathrm{ml}$ or less. Of these, 28 were positive to the $\mathrm{Pt}$ salts only, one to halide salts of Pt and Pd, one to

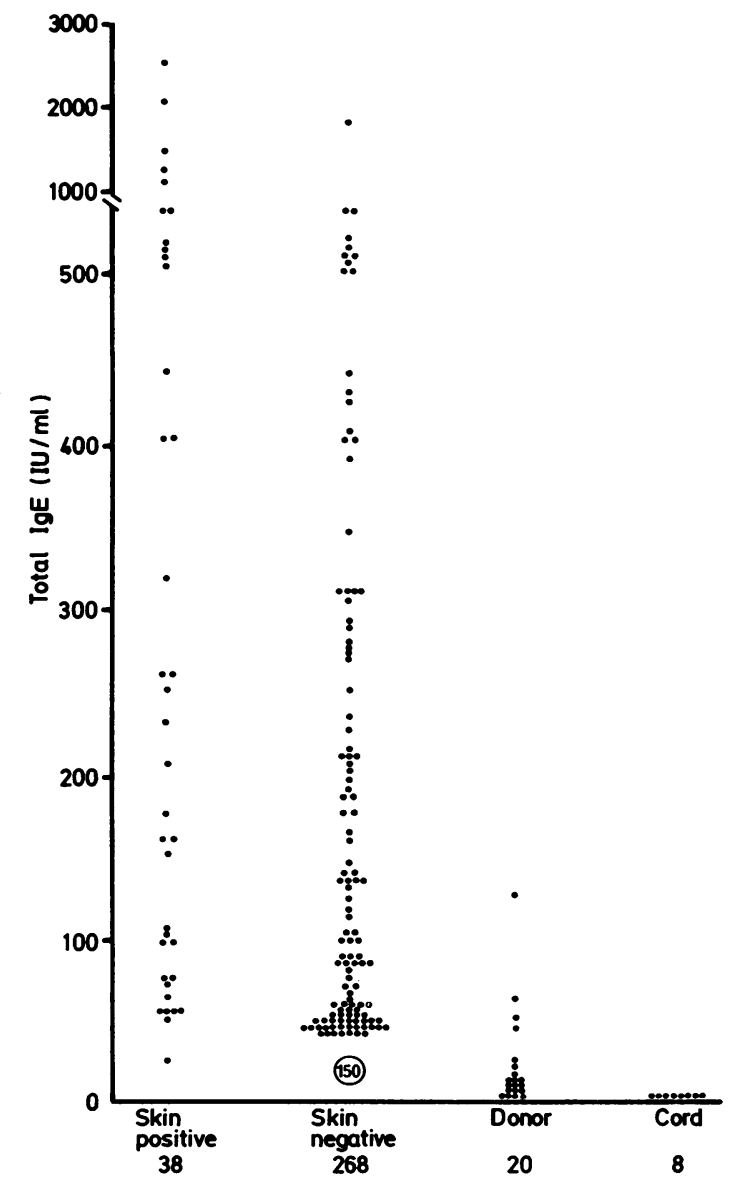

Fig 1 Total serum IgE in platinum salt skin test positive and skin test negative refinery workers, non-atopic blood donors, and cord blood sera. halide salts of Pt and Ru, two to halide salts of Pt and $\mathrm{Ir}$, one to halide salts of $\mathrm{Pt}$ and $\mathrm{Rh}$, one to halide salts of $\mathrm{Pt}, \mathrm{Ru}, \mathrm{Ir}$, and $\mathrm{Rh}$, and four to halide salts of $\mathrm{Pt}$, $\mathrm{Rh}$, and Ir. The remaining 268 were skin test negative. The 20 blood donors were not tested. None was atopic or had worked in a refinery or with precious metals.

TOTAL IgE

Figure 1 shows that 24 of the 38 skin test positive group had raised total serum IgE levels relative to the blood donor pool, with anthmetic mean values of 442 $\mathrm{IU} / \mathrm{ml}$ (SD 563 range $25-2500 \mathrm{IU} / \mathrm{ml}$ ) for the refinery population and $30.1 \mathrm{IU} / \mathrm{ml}$ (SD 43.3 range 5-150 $\mathrm{IU} / \mathrm{ml}$ ) for the control pool respectively.

The reason for the raised IgE levels in the platinum salt skin test positive group is not known. There is evidence in animals that IgE levels, both total and specific, are raised in adjuvant ( $B$ pertussis) primed animals with chronic exposure to platinum salts, as

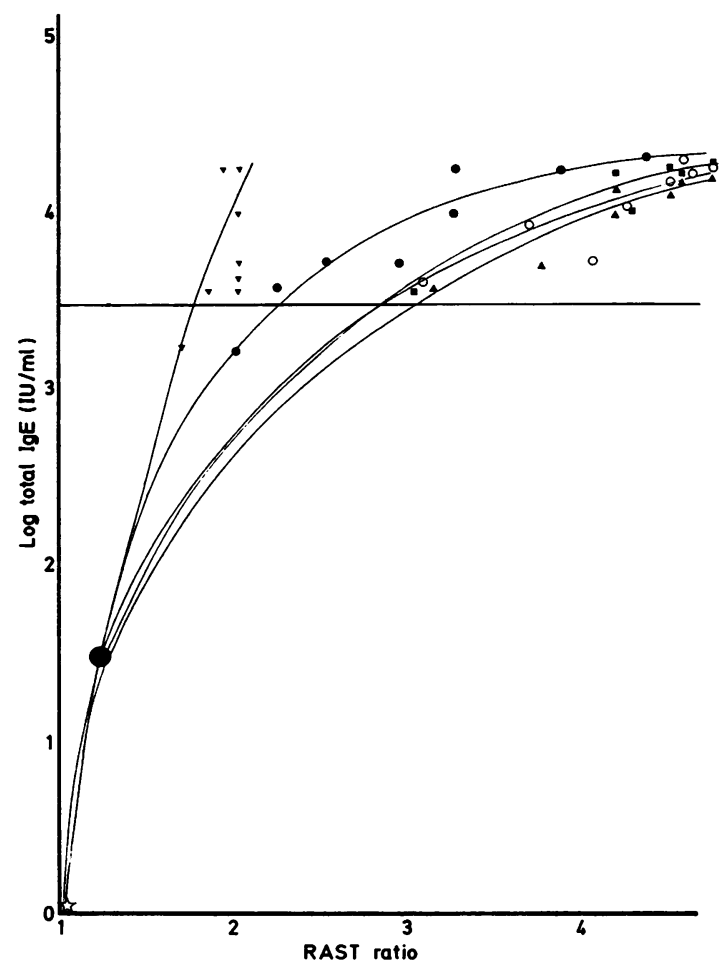

Fig 2 Non-specific interference of pooled cord blood ( pooled non-atopic donor sera ( ) and eight Hodgkin's patients' sera with differing levels of serum IgE (1750-22,500 $\mathrm{iu} / \mathrm{ml}$ ) in the five RAST systems. (O) platinum, (V)

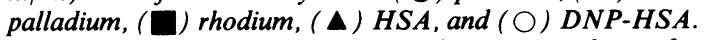
Solid horizontal line at $3000 \mathrm{iu} / \mathrm{ml}$ indicates upper limit of total IgE interference by test sera in various systems. 
opposed to the effects of adjuvant alone. ${ }^{19}$ It has also been found that total IgE levels are raised in platinum sensitive workers as compared with non-sensitive workers. ${ }^{13}$

\section{NON-SPECIFIC IgE UPTAKE}

Eight cord sera were tested in all assays to determine the lowest level of non-specific uptake. As a result of these findings subsequent readings were expressed as a RAST ratio relative to the cord levels:

RAST ratio $=\frac{\text { Sample cpm }- \text { background cpm }}{\text { Mean cord cpm }- \text { background cpm. }}$

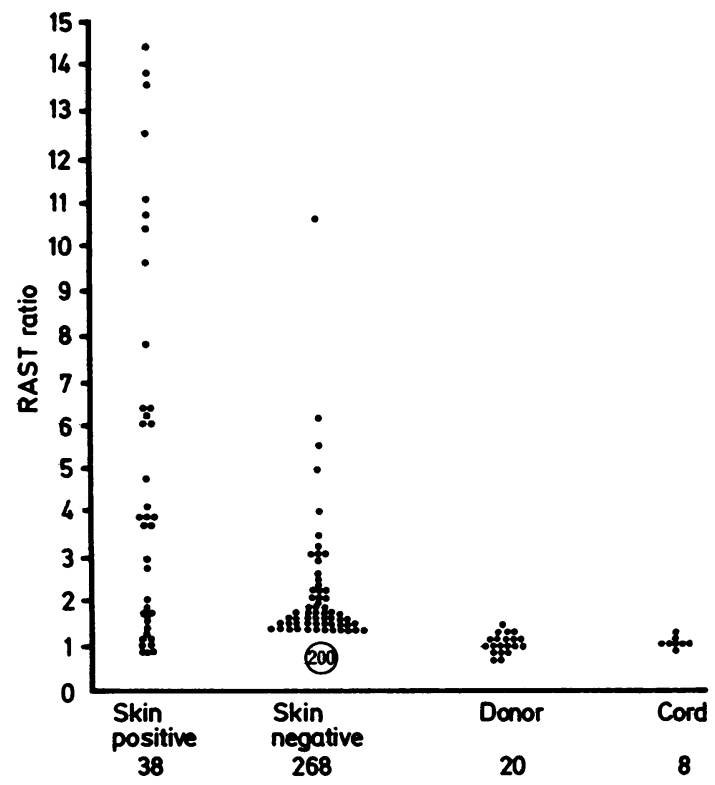

Fig 3 Platinum RAST in platinum salt skin test positive and negative subjects, non-atopic donor sera, and cord blood.

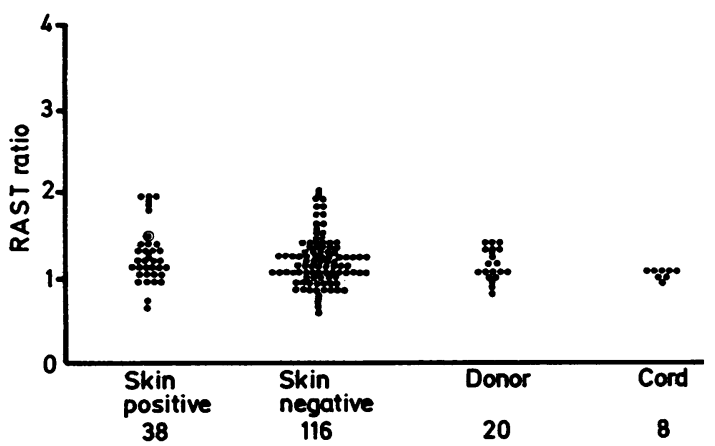

Fig 4 Palladium RAST in platinum salt skin test positive and negative subjects, non-atopic donor sera, and cord blood. (O) denotes palladium salt skin test positive subject.
Figure 2 shows the RAST ratios for the cord pool, blood donor pool, and the individual patients with Hodgkin's disease. Pooled cord sera by definition $\overline{\overline{\widehat{D}}}$ gave a value of 1 unit. Pooled donor sera gave a mean value of 1.2 units in all systems and those of the $\bar{C}$ patients with total IgE levels above $10000 \mathrm{IU} / \mathrm{ml}$ gave values of 4.0 units or more.

None of the total IgE levels for the refinery group was greater than $2500 \mathrm{IU} / \mathrm{ml}$, thus an arbitrary designated cut off ratio for non-specific binding at $\vec{O}$

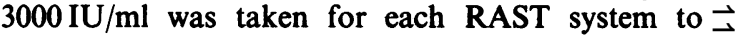
exclude any possible interference due to high IgE lev- $\omega$ els. The ratios taken as probably positive for each $O$ system was as follows: platinum $2 \cdot 1$, rhodium $2 \cdot 6$, pal- $\frac{\mathbb{}}{3}$ ladium 1.8, HSA 3.0, and DNP-HSA $2 \cdot 8$.

PLATINUM SALT RAST (fig 3)

All 306 sera were assayed blind for platinum salt antibody levels and subsequently separated into skin test $\frac{}{5}$ positive and skin test negative groups. Of the 38 skin $\vec{c}$ positive group, $23(62 \%)$ had ratios greater than the cut off point of $2 \cdot 1$ units, many sera giving ratios 10 -fold greater than the cord levels. By contrast only $16(6 \%)$ of the 268 platinum skin test negative group $\overrightarrow{0}$ had ratios greater than $2 \cdot 1$ units. The donor control $\infty$ pool ratio was $1 \cdot 2$.

PALLADIUM SALT RAST (fig 4)

The RAST ratios found in the 38 platinum salt skin test positive group were no different from the selected $\stackrel{0}{\circ}$

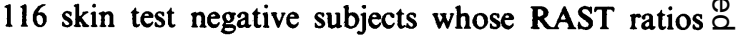
were equal to or greater than unity. No values greater $\overrightarrow{\overrightarrow{0}}$ than 2 units were observed; this correlated well with $\exists$ the presence of only one skin test positive reaction to the palladium salt. This worker gave a $2 \mathrm{~mm}$ weal at $10^{-5} \mathrm{~g} / \mathrm{ml}$ and a RAST ratio of 1.35 units. Donor blood samples gave values close to unity.

RHODIUM RAST (fig 5)

Of the 38 platinum salt skin test positive subjects, six $\delta$ also had positive reactions to the rhodium salt. Four $₹$ of the six also had RAST scores greater than 2.6; only 0 five of the 116 platinum salt skin test negative $(4 \%)$ had values greater than this.

HSA RAST (fig 6)

Taking the ratio of 3 as the probable criterion of pos- $N$ itivity, the incidence of significant binding to the HSA solid phase was low in all groups. Four (10.5\%) of the 38 platinum salt skin test positive group and only three $(2.5 \%)$ of the 116 platinum salt negative group $\underset{\Phi}{\bar{D}}$ gave positive results.

DNP-HSA RAST (fig 7)

The conjugation of DNP to HSA to form anti- $\underset{\mathbb{D}}{\mathbb{D}}$ genically denatured HSA produced greater numbers 
of positive results in the platinum salt sensitive group, eight of the $38(21 \%)$ having ratios greater than the non-specific uptake level of $2 \cdot 8$. Of these eight, four had positive RASTs to HSA. Positive RASTs to the rhodium salt were obtained in four of this group, and of these, two had positive RASTs to HSA and four to DNP-HSA. Positive RASTs were obtained with the rhodium salts only with sera from platinum salt positive subjects. In the platinum salt skin test negative group only four of the $116(3.4 \%)$ had positive RASTs to DNP-HSA and three of these gave positive HSA RASTs. All four platinum salt skin test positive

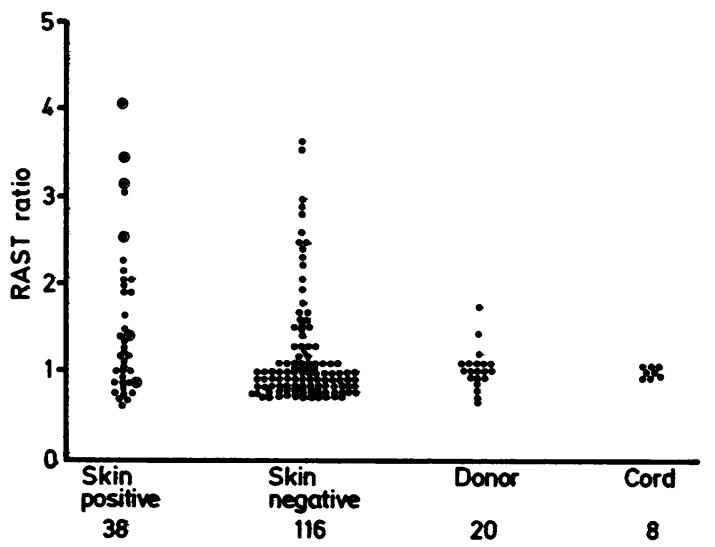

Fig 5 Rhodium RAST in platinum salt skin test positive and negative subjects, non-atopic donor sera, and cord blood. (O) denotes rhodium salt skin test positive subjects.

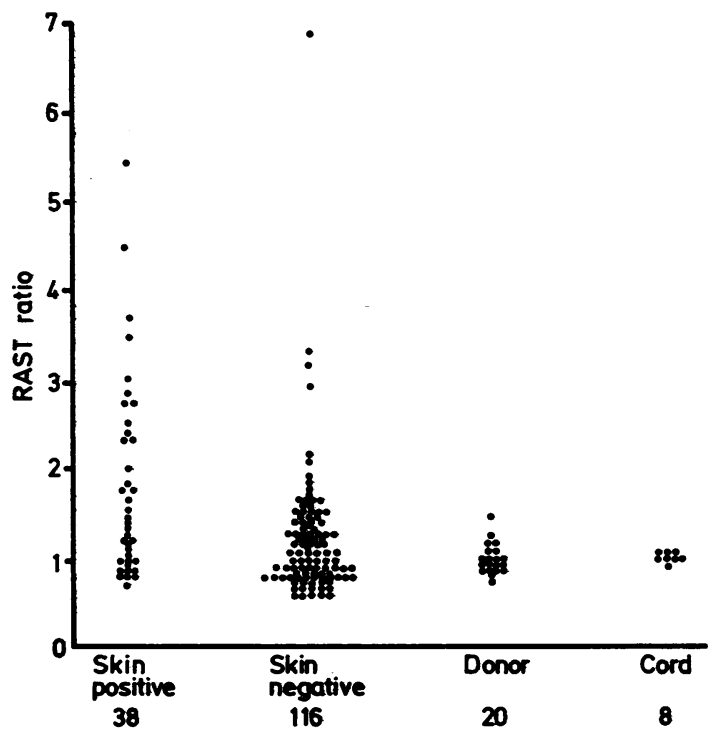

Fig 6 Human serum albumin (HSA) RAST in platinum salt skin test positive and negative subjects, non-atopic donor sera, and cord blood. subjects with raised HSA Rasts also had positive DNP-HSA RAST scores. Four of the eight platinum salt sensitive group with positive DNP-HSA RAST also had positive rhodium salt RAST.

\section{CORRELATION OF TOTAL SERUM IgE LEVELS \\ AND RAST RATIO}

The sera of the patients with Hodgkin's disease with greatly raised total IgE levels gave positive RAST scores in all five systems. Examination of the RAST scores and total IgE levels in the refinery workers using Spearman's rank correlation test gave the following results.

Total IgE $v$ platinum-HSA RAST, $\mathrm{r}=\mathbf{0 . 5 2}$

Total IgE $v$ rhodium-HSA RAST, $r=0.31$

Total IgE palladium-HSA RAST, $r=0 \cdot 18$

Total IgE $v$ HSA RAST, $\mathrm{r}=0.36$

Total IgE $v$ DNP-HSA RAST, $\mathrm{r}=\mathbf{0 . 3 4}$

It may be concluded, therefore, that whereas greatly raised total IgE levels, as found in the Hodgkin's sera, significantly increase RAST scores, this is not the case with the RASTs for palladium, rhodium, HSA, and DNP-HSA. With regard to the total IgE Pt-HSA comparisons there was some correlation. PtHSA RASTs (fig 3), however, show much higher RAST values for many sera with total IgE levels not exceeding 2500 units as compared with the values for the Hodgkin's sera when many sera were in excess of 10000 units.

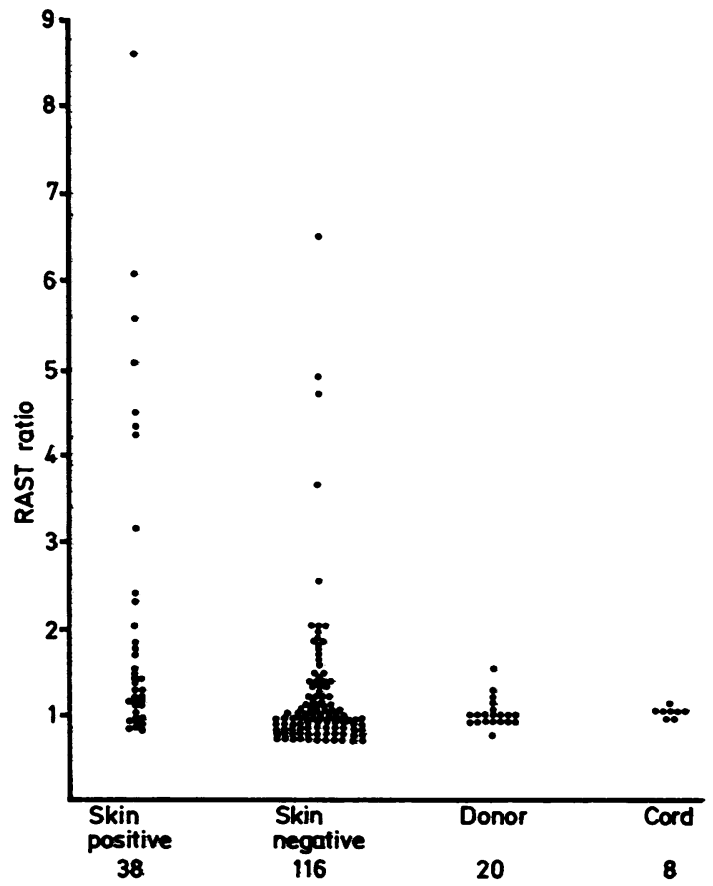

Fig 7 DNP-HSA RAST in platinum salt skin test positive and negative subjects, non-atopic donor sera, and cord blood. 


\section{Discussion}

The potent allergenicity of the halide salts of platinum in refinery conditions is well documented and the causal agents have been identified as the divalent and tetravalent platinum compounds possessing reactive halide ligands-namely, ammonium tetrachloroplatinite and ammonium hexachloroplatinate. ${ }^{3}$ Their mode of action is thought to be by conjugation with sulphydryl containing groups within proteins, thus forming immunogenic complexes. It might be expected that other group VIII metal salts-for example, palladium and rhodium-which possess similar physicochemical characteristics in terms of their size, charge, and protein reactivity would be capable of firm conjugation with proteins in vivo to form stable complexes, and that these conjugates in vivo should be capable of inducing antibody formation and hypersensitivity. Past studies, however, have shown that other group VIII metal salts are not potent allergens. The present study of platinum salt positive prick tests and positive RASTs shows moderately good concordance, and also shows that the other platinum group metal salts are poor sensitisers as shown by skin prick test (at non-histamine releasing concentrations) or the RAST. Of the 306 workers, only six had positive skin tests to the rhodium salt and only one had a positive prick test to the palladium salt. Interestingly, there were no cases of positivity to these salts in the absence of a platinum salt skin test reaction.

There is some evidence to suggest that in some workers limited cross reactivity between the salts of platinum and palladium salts might be present in sensitised workers. This evidence comprises: (1) the presence of palladium salt skin prick positivity which has been noted in platinum salt sensitised workers, with no reports of isolated type 1 hypersensitivity to palladium salts; (2) PCA reactions in the monkey which have been obtained to the palladium salt only with the sera of platinum sensitive workers; and (3) PCA cross absorption studies in the monkey (R D Murdoch, in preparation) which have failed to detect specific palladium salt antibodies when the platinum salt antibody activity has been absorbed, and that the palladium salts can also absorb though not completely the platinum salt activity. RAST inhibition studies (R D Murdoch, personal communication) using the same subject as in (3) corroborate these findings.

In tests for antibodies against new antigenic determinants in HSA, which is a probable carrier protein by virtue of its high concentration in body fluids, the incidence of significant RAST binding in the HSA RAST system showed low levels, with only $2.5 \%$ of the 116 platinum skin test negative subjects tested being positive, whereas $10.5 \%(4 / 38)$ of the platinum sensitive subjects were positive. The presence of $\operatorname{IgE} \frac{\mathbb{Q}}{\mathbb{Q}}$ antibodies against denatured HSA in $21 \%$ of those possessing IgE antibody against the platinum salts, $\stackrel{\vec{\rho}}{\rightarrow}$ compared with only $3.4 \%$ in the skin test negative group, suggests that the HSA is behaving as a partial of antigenic determinant in its denatured form.

In both the DNP and the platinum salt RAST solid $\bar{\varnothing}$ phase the degree of haptenic substitution was 6.1 and 8 . Thus with similar substitutions the question arises as to where the haptens are conjugated. DNP-HSA $\vec{\circ}$ was taken, arbitrarily, as an example of denatured $\vec{\overrightarrow{ }}$ HSA in tests for antibodies to new antigenic deter- $\omega$ minants on HSA. DNP will preferentially bind to $\bar{\circ}$ lysine residues and terminal amino groups, whereas 3 the platinum halide salts are preferentially bound to $\vec{c}$ the sulphydryl groups of methionine, cysteine, and histidine. Those that are accessible are, however, lim- $\dot{\omega}$ ited to fewer than the number of bound platinum 0 molecules and the possibility of the highly charged platinum complex penetrating the hydrophobic inte- $\vec{c}$ rior of the HSA is questionable; this suggests that binding must occur elsewhere. It is not known, however, whether the conformational changes brought $₹$ about by DNP and the platinum salts would produce $\vec{\varphi}$ sufficiently similar changes to allow binding of closely related antibody. The ideal control would be a heavy metal salt-HSA interaction, but it was thought that a denatured HSA unrelated to the effects of such salts was preferable. The positive RASTs to DNP-HSA would support the assumption that HSA is acting as $\frac{2}{\varnothing}$ a carrier molecule for the haptenic platinum salts and $\cong$ that a denatured site is produced within the HSA $\overrightarrow{\overrightarrow{0}}$ molecule capable of binding specific IgE antibody. 3 This denaturation may be at sites common to the platinum, palladium, and rhodium salts and to DNP, accounting for the large numbers of positive responses. The possibility of specific hypersensitivity $\frac{\mathbb{S}}{3}$ to the other platinum group metals cannot be excluded, though the evidence shows that this is unlikely. Cross reactivity based on antibodies to 8 denatured HSA, which could readily be produced by these metals, provides an alternative explanation for 0 reactions to them occurring in the platinum sensitive $\rightarrow$ subjects.

High total serum IgE levels are known to interfere with all RAST systems so there are always problems or of deciding the criteria for a positive result. Thus by $N$ choosing a RAST cut off point greater than any effect N which could be caused by non-specific high total IgE $\sigma$ interference, the test is more useful for screening large numbers of sera without the need for dilution of $\frac{0}{0}$ serum samples. Diluting the sera $1 / 10$ is known to $\stackrel{\oplus}{?}$ result in $60 \%$ reduction in platinum salt RAST bind- 0 ing (unpublished data). In recent investigations the presence of antibody to other platinum group metal $\stackrel{\vec{D}}{\circ}$ salts, in particular to palladium, has been reported, ${ }^{16} \frac{\rho}{\mathbb{D}}$ 
but could not be confirmed in a later study with the same sera (RD Murdoch, unpublished data).

Soviet investigations have reported type 1 hypersensitivity in rodents and rabbit to most group VIII metal salts and to other heavy transition metal salts. ${ }^{17-19}$ Many of their protocols have been repeated in attempts to induce or to elicit type 1 reactions without success. ${ }^{1520}$ At these specified doses for eliciting reactions, the histamine liberating properties of the platinum salts have played a major pharmacological part. ${ }^{21}$

We thank Johnson Matthey plc for providing the platinum group metal salts and their generous financial assistance throughout the duration of this work, Dr P Amlot for providing the Hodgkin serum samples, and Miss C Langan for preparing the manuscript.

\section{References}

${ }^{1}$ Hunter D, Milton R, Perry KMA. Asthma caused by the complex salts of platinum. Br J Ind Med 1945;2:92-8.

${ }^{2}$ Roberts AE. Platinosis: a five year study of the effects of soluble platinum salts on employees in a platinum laboratory and refinery. Archive of Industrial Hygiene and Occupational Medicine 1951;4:549-55.

${ }^{3}$ Cleare MJ, Hughes EG, Jacoby B, Pepys J. Immediate (type 1) allergic responses to platinum compounds. Clin Allergy 1979;9:109-17.

${ }^{4}$ Sheard C. Contact dermatitis from platinum and related metals. Archives of Dermatology 1955;71:357-60.

${ }^{5}$ Lido-Dunipe E. Sensibilization al platino per soldodura. Acta Dermo (Madrid) 1957;48:543-84.

${ }^{6}$ Munro-Ashman D, Munro D, Hughes TH. Contact dermatitis from palladium. Transactions of the St John's Hospital Dermatological Society 1969;55:196-7.

${ }^{7}$ Howe W, Venables KM, Topping MD. Tetrachlorophthalic anhydride asthma: evidence for specific IgE antibody. J Allergy Clin
Immunol 1983;71:5-11.

${ }^{8}$ Bernstein DI, Patterson R, Zeiss CR. Clinical and immunological evaluation of trimellitic anhydride and phthalic anhydrideexposed workers using a questionnaire with comparative analysis of enzyme linked immunoabsorbent and radioimmunoassay studies. J Allergy Clin Immunol 1982;69:311-8.

${ }^{9}$ O'Brien IM, Harries MG, Burge PS, Pepys J. Toluene diisocyanate asthma: I reactions to TDI, MDI, HDI, and histamine. Clin Allergy 1979;9:1-6.

${ }^{10}$ Zeiss CR, Levitz D, Chacon R, Wolkonsky P, Patterson R, Pruzansky JJ. Quantitation and new antigenic determinant (NAD) specificity in antibodies induced by inhalation of trimellitic anhydride in man. Int Arch Allergy Appl Immunol 1980;61:380-8.

${ }^{11}$ Dolovich J, Evans SH, Nieboer E. Occupational asthma from nickel sensitivity: 1 human serum albumin in the antigenic determinant. Br J Ind Med 1984;41:51-5.

${ }^{12}$ Nieboer E, Evans SL, Dolovich J. Occupational asthma from nickel sensitivity: II factors influencing the interaction of $\mathrm{Ni}^{2+}$, HSA, and serum antibodies with nickel related specificity. $\mathrm{Br} J$ Ind Med 1984;41:56-3.

${ }^{13}$ Cromwell O, Pepys J, Parish WE, Hughes EG. Specific IgE antibodies to platinum salts in sensitised workers. Clin Allergy 1979;9:109-17.

${ }^{14}$ Hudson L, Hay F. Practical immunology. Oxford: Blackwell Scientific Publications, 1980.

${ }^{15}$ Murdoch RD. Immunological responses to complex salts of platinum. London: University of London. 1982. (PhD thesis.)

${ }^{16}$ Biagini RE, Clark JC, Gallagher JS, Bernstein K, Moorman WM. Passive transfer in the monkey of human immediate hypersensitivity to complex salts of platinum and palladium. Fed Proc 1982; July, 3255.

${ }^{17}$ Tomilets VA, Dontsov VI, Zakharova IA. Immediate and delayed allergic reactions to group VIII metals in an experiment. Fiziol Z 1979;25:653-7.

${ }^{18}$ Tomilets VA, Zakharova IA. Anaphylactic and anaphylactoid properties of palladium complexes. Farmakol Toksikol 1979;42:170-3.

${ }^{19}$ Tomilets VA, Dontsov VI, Zakharova IA, Klevtsov K. Histamine releasing and histamine binding actions of platinum and palladium compounds. Archivum Immunologiae et Therapiae Experimentalis (Warszawa) 1980;28:953-60.

${ }^{20}$ Murdoch RD, Pepys J. Immunological responses to complex salts of platinum I specific IgE antibody production in the rat. Clin Exp Immunol 1984;57:107-14.

${ }^{21}$ Saindelle A, Ruff F. Histamine release by sodium chloroplatinate. Br J Pharmacol 1969;35:313-21. 